\title{
A Purely Mechanical Energy Storing Concept for Hybrid Vehicles
}

\author{
Latchezar Tchobansky \\ Martin Kozek \\ Gerd Schlager \\ Hanns P. Jörgl
}

Institute for Machine- and Process-Automation, Vienna University of Technology

Copyright @ 2003 SAE International

\begin{abstract}
The paper contains the design and simulation of a purely mechanical system for storing energy during vehicle deceleration, which can be utilized during subsequent acceleration (regenerative braking). A continuously variable transmission (CVT) regulates the energy transfer, energy storage is accomplished by means of a spiral spring and additional use of a planetary transmission. The transmission ratio of the CVT is adapted by a combined feed-forward and feed-back control. A computer simulation for a vehicle with an overall mass of $1500 \mathrm{~kg}$, and an energy storage capacity of $30 \mathrm{~kJ}$ was performed for different driving cycles. In a typical urban stop and go situation the proposed device will save up to $0.72 \mathrm{~kg}$ fuel per $100 \mathrm{~km}$ and the overall efficiency analysis together with simple design, easy recycling, and long life expectance, showed a clear advantage over other hybrid concepts for frequent stops and accelerations.
\end{abstract}

\section{INTRODUCTION}

The continuously increasing demand for individual mobility and the emerging implications on society, environment and economics are a perpetual motivation for developing and investigating alternative low emission propulsion concepts. Today an estimated 500 millions of passenger cars are in use, most in them in urban areas [1]. Not only the goal of an efficient use of resources but also the local air pollution caused by vehicle emissions form a strong incitement for legislative conditions. Reducing the adverse effects on the environment and meeting the legislative requirements is imperative but doing this with maximum economic efficiency is also dictated by any responsible design.

Vehicle engines used in urban areas with low average travel velocities, frequent stops, and idling periods have overall efficiencies clearly below $10 \%$ [2]. The energy necessary for propulsion is not only consumed to overcome the cumulative rolling resistances but a considerable part of energy is required for acceleration. During typical 'stop-and-go' stretches this acceleration energy is dissipated through the main brakes immediately afterwards. Additionally, the vehicle engine is almost always operating under sub-optimal working conditions leading to high emissions, high wear, and low efficiency.

In order to overcome or at least diminish these problems part of the kinetic energy may be accumulated during deceleration phases, stored in a proper device, and used for a subsequent acceleration. This procedure is also termed as "regenerative braking" since each deceleration event replenishes the stored energy used for acceleration. Due to friction and other irreversible processes like battery charging losses are always present and additional energy from the main engine will always be necessary during strong or longer lasting accelerations. Likewise, the power flow during energy accumulation will be limited and the main brakes are still needed during braking maneuvers.

The necessity to provide a positive torque to the drive train fed from the stored kinetic energy calls for a second engine inside the vehicle. This concept with two different sources of propulsion inside a vehicle is generally called hybrid.

In most cases the idea of hybrid cars is associated with a combination of an internal combustion engine with an electric drive. This concept allows merging the advantages of both engines paying a price of additional weight and higher complexity. Although a considerable reduction of emissions especially in urban areas with heavy traffic load is possible with this concept, regenerative braking has low efficiency mostly due to the charging/discharging characteristics of the batteries. 
The main idea presented in this paper is the use of a purely mechanical device for regenerative braking. Several mechanical devices have been proposed for the same purpose. Among them the original idea of using spiral springs was already filed for patent in 1976 [3]. Also rubber torsion springs together with a variable belt transmission have been patented [4], and planetary transmissions connected to friction clutches have been suggested [5]. A concept using a continuously variable transmission (CVT) together with a planetary gear but utilizing a flywheel for energy storage has also been proposed [6].

The concept proposed in this paper is comprised of similar components, but the arrangement is modified in order to guarantee a well defined energy flow from and to the storage device under a wide range of driving conditions. The CVT regulates the energy transfer during braking or acceleration, and energy storage is accomplished by means of a spiral spring and additional use of a planetary transmission. The transmission ratio of the CVT is adapted by computer control which takes the drivers requests as inputs and decides how the power flow is balanced between engine, storage device, and brakes. The device is comparatively light-weight, compact and designed using standard automotive components already in use today.

In the remainder of the paper a detailed description of the mechanical storage device will be given including transmission ratio calculations for important operating states. Special attention will be paid to the technical feasibility of the mechanism and ensure proper function under a wide range of driving conditions. The control concept with a combined feed-forward and feed-back algorithm is also addressed, and a section on simulation results indicates the potential of the mechanism for energy saving. The paper is concluded by some statements on the remaining problems and future work.

\section{MAIN SECTION}

\section{MECHANISM}

The purely mechanical device for energy storage consists of 3 main parts:

- A torsion (spiral) spring,

- a planetary transmission,

- a continuously variable transmission (CVT).

As depicted in Figure 1, the planetary transmission and the CVT constitute a specialized unit since a conventional transmission runs at a fixed ratio parallel to the CVT. Both the CVT (2CVT) and the conventional gear (Z021) run with the rotational speed $\mathrm{n} 2$ of the input shaft. The second disc of the CVT (1CVT) is in rigid combination with the sun gear (z1) of the planetary transmission, and the second gear (Z021) of the conventional transmission is fixed with the ring gear ( $\mathrm{z} 3$ ) of the planetary transmission.

One end of the storage spring is fixed at the housing of the device, the other end is fixed at the carrier (n5) of the planetary gear. The whole device can be separated from the drive train using a conventional clutch (K2), which will only be operated in special situations (energy storage for long time). During normal driving situations it may be utilized as a safety device.

If the vehicle is moving all components of the device will also move, however, the direction and speed of rotation of the carrier (and consequently the question if the carrier moves at all) is determined by the transmission ratio of the CVT. Therefore, by adjusting the transmission ratio of the CVT energy can be either accumulated in the storage spring, or stored there for nearly infinite time, or it may be utilized for accelerating the vehicle.

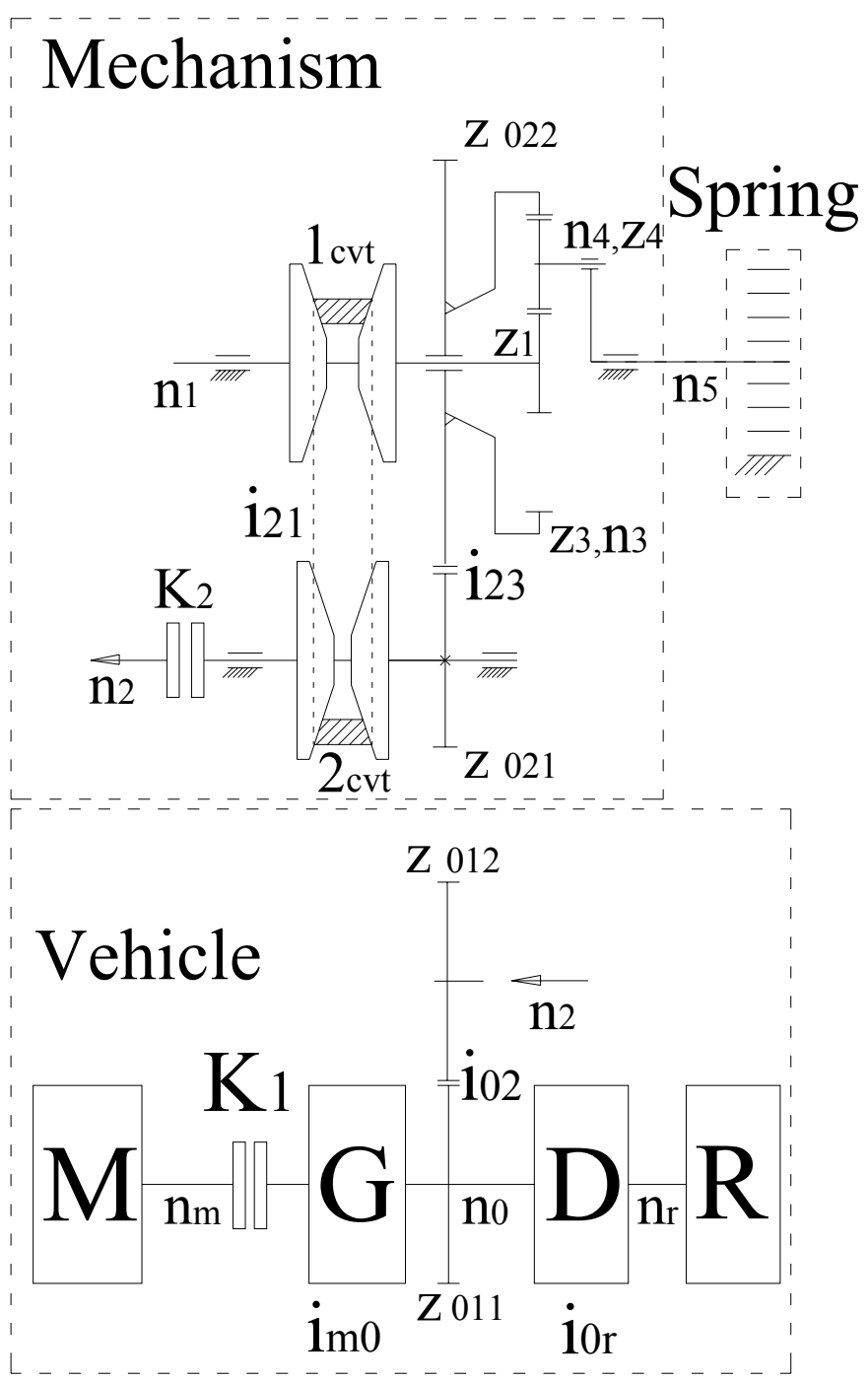

Figure 1: Schematic of the energy storage device and the drive train. 
The vehicle is lumped into 5 main parts: Engine $(\mathrm{M})$, clutch (K1), gear box (G), differential gear (D) and wheels $(R)$. The input shaft to the device (no) is coupled to the drive train between gear box and differential gear.

The most problematic part of the transmission design is the correct choice of the planetary transmission. This is due to the fact that planetary gears themselves have several geometric restrictions for reliable and safe operation, and in this application additional interactions with the CVT and the spiral spring pose further restrictions.

Since the proposed mechanism is still a concept and a feasibility study rather than a rigorous design, no detailed investigations on strength or life expectance of the various components have been performed yet. Hence, the geometric data of the device have been mainly chosen based on kinematic considerations, although data from implemented similar transmissions have also been incorporated.

Important data of the components necessary for the simulation are given in the section Simulation Results.

\section{BASIC PRINCIPLE}

A change of the system characteristics is achieved by adjusting the CVT transmission ratio. Different speed ratios between the CVT shafts will determine if energy is stored in the device or taken for acceleration.

\section{Energy accumulation}

In order to accumulate energy, part of the kinetic energy of the vehicle should be transferred into an elastic deformation of the spring. This is achieved by adjusting the CVT ratio such that a rotation of the carrier of the planetary gear results. If this rotation is directed against the momentum of the spring additional energy is stored and the spring is further wound up.

This storage concept allows the application of a wide range of decelerating torques on the vehicle, however, the total amount of energy stored in the spring is limited, and also the dead time between operation of the brake pedal and CVT adjustment is much to large for critical driving situations. In these cases the main brakes have to be additionally used.

Due to opposed inner moments in the planetary gear bracing will result and the friction losses (Pv) inside the transmission will increase. These inner losses show a nonlinear proportionality to the momentum of the spring.

\section{Energy consumption}

This is the opposite case where potential energy from the spring is utilized to accelerate the vehicle or, in the case of constant speed, to compensate the additional friction losses mentioned above. The transmission ratio of the CVT is now chosen in a way that the rotational direction of the carrier is reversed. Since rotation and spring torque have the same direction in this setup, energy is extracted from the storage device. Again, if the amount of energy stored is too small or the power needed for acceleration is larger than provided by the CVT additional torque has to be provided by the vehicle engine.

\section{Energy storage}

During stopping, parking, or coasting no torque is required in the drive train. In this situation the additional clutch separates the device completely from the drive train and the transmission ratio of the CVT is chosen to simply lock the spring in the original position. In this way stored spring energy can be conserved virtually without loss and additional noise as opposed to solutions utilizing a battery or a flywheel.

The unusual combination of coupled CVT, spring, planetary and conventional transmission requires a thorough geometrical analysis. In order to quantitatively define the correct transmission ratio of the CVT all these components have to be considered.

\section{TRANSMISSION RATIOS}

The central problem for the correct choice of the transmission ratio of the CVT is the definition and computation of the freewheel ratios i21f and i21d. The transmission ratio i21f will be called static freewheel ratio and $\mathrm{i} 21 \mathrm{~d}$ is defined as the dynamic freewheel ratio.

The static CVT freewheel transmission ratio (i21f) is solely determined by the geometric design of the planetary and conventional gear. If this ratio is applied to the CVT the carrier remains static $\left(n_{5}=0\right)$, and since no rotational motion of the spring is possible no energy can be transferred to or from the spring. In this case the torque transfer between spring and vehicle is also zero.

The dynamic CVT freewheel transmission ratio (i21d) takes into account the additional friction losses due to bracing. As mentioned earlier, this situation arises during constant speed stretches with energy stored in the device. This transmission ratio additionally depends on the vehicle state (speed, drive train torque, stored spring energy) and should exactly compensate the additional losses due to the storage device. In this way unwanted rolling resistance is eliminated by design.

Instead of applying the dynamic freewheel ratio i21d separating the whole storage device from the drive train would also eliminate additional losses. Apparently, if the spring is completely unwound i $21 \mathrm{f}$ and i $21 \mathrm{~d}$ are identical

\section{Static CVT freewheel transmission ratio (i21f)}

The computation of the static CVT freewheel transmission ratio is done as the first part. For the static transmission ratio of the planetary gear $(\mathrm{u})$ the following holds [4] (see also Figure 1): 
$\mathrm{u}=-\frac{\mathrm{z}_{3}}{\mathrm{z}_{1}}$

$\mathbf{Z}_{1}, \mathrm{Z}_{3}$ - number of gear teeth for inner and outer planetary gear

The rotational speeds of the individual components (inner gear $\left(n_{1}\right)$, outer gear $\left(n_{3}\right)$, and carrier $\left(n_{5}\right)$ ) of the planetary transmission show the dependency

$\mathrm{n}_{1}=\mathrm{u} \cdot \mathrm{n}_{3}+(1-\mathrm{u}) \cdot \mathrm{n}_{5}$

The rotational speed at the input shaft of the CVT is given by

$\mathrm{n}_{2}=\frac{\mathrm{n}_{0}}{\mathrm{i}_{02}}$,

and the individual speeds of the planetary components can thus be derived as

$\mathrm{n}_{1}=\frac{\mathrm{n}_{2}}{\mathrm{i}_{21}}=\frac{\mathrm{n}_{0}}{\mathrm{i}_{02} \cdot \mathrm{i}_{21}}$

$\mathrm{n}_{3}=\frac{\mathrm{n}_{2}}{\mathrm{i}_{23}}=\frac{\mathrm{n}_{0}}{\mathrm{i}_{02} \cdot \mathrm{i}_{23}}$

$\mathrm{n}_{5}=\frac{\mathrm{n}_{1}-\mathrm{u} \cdot \mathrm{n}_{3}}{1-\mathrm{u}}$

i02, i23 - transmission ratios

no - rotational speed of power train behind gear box

Combining all the above equations and solving for the rotational speed of the carrier $n_{5}$ as a function of the CVT transmission ratio i 21 leads to the equation

$\mathrm{n}_{5}=\frac{\mathrm{n}_{0}}{\mathrm{i}_{02} \cdot(1-\mathrm{u})} \cdot\left[\frac{1}{\mathrm{i}_{21}}-\frac{\mathrm{u}}{\mathrm{i}_{23}}\right]$

During normal driving conditions the carrier speed $\mathrm{n}_{5}$ can only vanish if the difference inside the brackets becomes zero. In this case the carrier remains static and a sufficient condition for the static freewheel transmission ratio is therefore:

$\frac{1}{i_{21 f}}=\frac{u}{i_{23}}$

Solving the above equation for i21f leads to the final formulation of the static CVT transmission ratio during freewheel operation: $\mathrm{i}_{21 \mathrm{f}}=\frac{\mathrm{i}_{23}}{\mathrm{u}}=\frac{\mathrm{z}_{1} \cdot \mathrm{z}_{022}}{\mathrm{z}_{3} \cdot \mathrm{z}_{021}}$

Z021, Z022 - number of gear teeth for conventional transmission

This geometric freewheel ratio is obviously somewhere in the middle of the range of the CVT transmission ratios, and from the formula (1) for the carrier speed $n_{5}$ it will be defined that:

$$
\begin{aligned}
& \mathrm{i}_{21}>\mathrm{i}_{21_{\mathrm{f}}} \Rightarrow \mathrm{n}_{5}<0 \quad \Rightarrow \text { energy accumulation } \\
& \mathrm{i}_{21}<\mathrm{i}_{21_{\mathrm{f}}} \Rightarrow \mathrm{n}_{5}>0 \Rightarrow \text { energy consumption }
\end{aligned}
$$

Obviously, $\mathrm{n}_{5}$ together with i21f constitutes the origin of the transmission ratios of the CVT, and by choosing different sets of gear teeth there are 2 degrees of freedom in the design of the mechanism:

1. The numbers of gear teeth $\mathrm{Z}_{1}, \mathrm{Z}_{3}, \mathrm{Z}_{021}, \mathrm{Z}_{022}$

2. The CVT freewheel ratio i21f

For practical reasons the CVT freewheel ratio i21f and the planetary gear teeth $\mathrm{Z}_{1}, \mathrm{Z}_{3}$ (and $\mathrm{Z}_{2}$ ) should be chosen as the best compromise and the conventional gear with $Z_{021}$, Z022 should then be fitted accordingly.

The sensitivity of the carrier speed $n_{5}$ with respect to the CVT transmission ratio i21 is given by differentiating equation (1) with respect to i21:

$\frac{\partial \mathrm{n}_{5}}{\partial \mathrm{i}_{21}}=\frac{\mathrm{n}_{0}}{\mathrm{i}_{02} \cdot(1-\mathrm{u})} \cdot \frac{1}{\mathrm{i}_{21,0}^{2}}$

Obviously, by a proper choice of i02 and $u$ the rate at which the carrier changes its speed can be adjusted. This means that the rate at which the mechanism reacts to a control input can be designed independently from the freewheel ratio i21f. From Figure2d, and the above formula it can be seen that for transmission ratios i21 close to i $21 \mathrm{~min}$ the sensitivity is largest which in turn is favorable for the initial acceleration phase.

For the overall transmission ratio i05 equation (1) can be rearranged and

$\mathrm{i}_{05}=\frac{\mathrm{n}_{0}}{\mathrm{n}_{5}}=\frac{\mathrm{i}_{02} \cdot \mathrm{i}_{21} \cdot \mathrm{i}_{23} \cdot(1-\mathrm{u})}{\mathrm{i}_{23}-\mathrm{u} \cdot \mathrm{i}_{21}}$

holds.

$\underline{\text { Considerations on the range of CVT transmission ratios }}$

In order to utilize the mechanism in a wide range of driving situations the range of CVT transmission ratios should be split up equally between acceleration and 
deceleration. Consider Figure 2a, where a sketch of the planetary gear can be seen.

In the depicted situation the driving shaft runs with constant speed $\left(\mathrm{n}_{2}=\right.$ const $)$ and the CVT is operating at a static freewheel transmission ratio of $\mathrm{i}_{21 \mathrm{f}}=1$ (hence, $\left.\mathrm{i}_{23}=\mathrm{u}\right)$. If the CVT transmission ratio is varied between the minimum (i21min) and the maximum (i21max) ratio, the carrier will rotate at different speeds depending on the

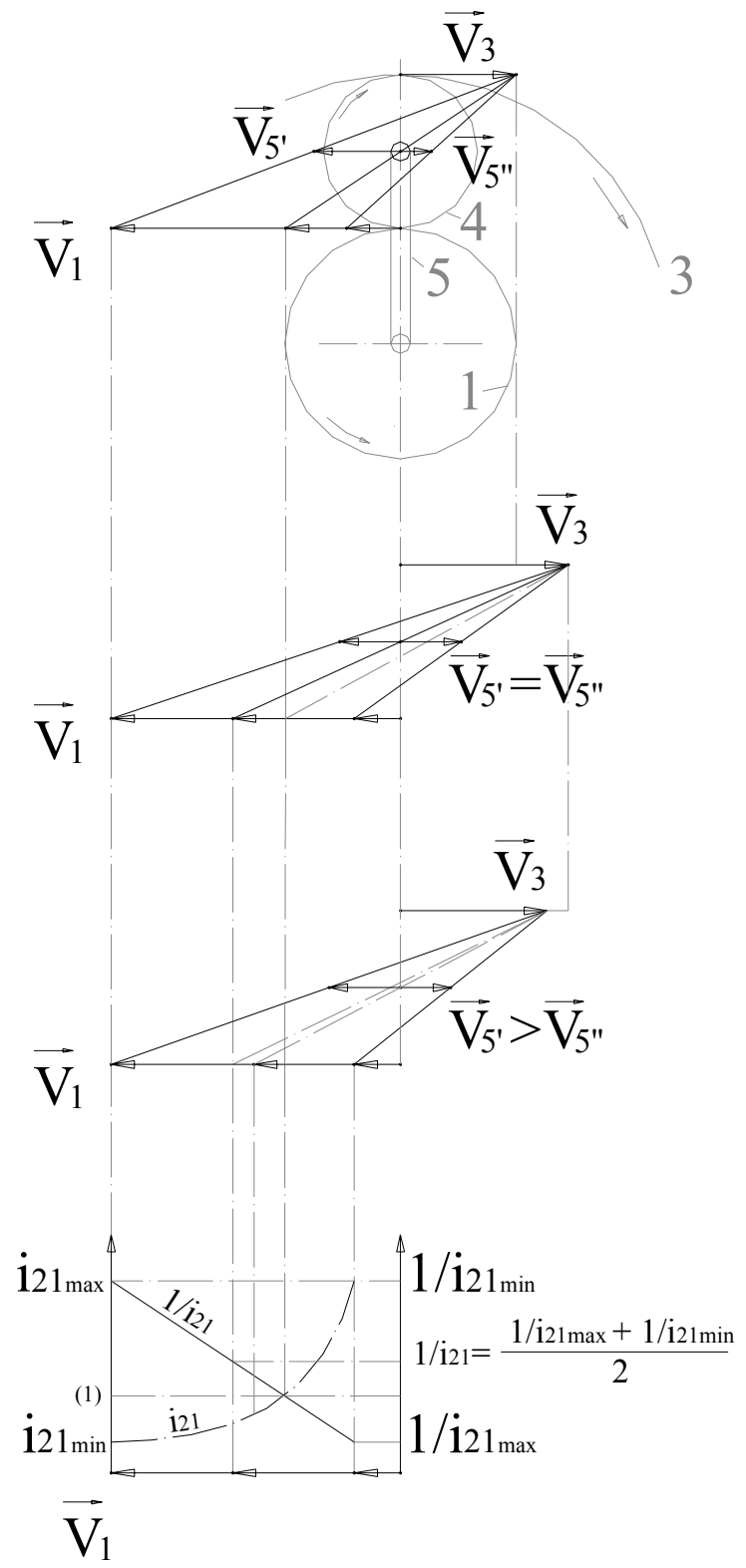

(a)

(b)

(c)

(d)

Figure 2: Contact point velocities in the planetary gear: 1 sun gear, 3 ring gear, 4 planet gear, 5 carrier
(a) $\quad \mathrm{i}_{21 \mathrm{f}}=1$
(a) $\quad \mathrm{i}_{21 \mathrm{f}}=\frac{2}{\mathrm{i}_{\text {min }}+\mathrm{i}_{\max }}$
(a) $\quad \mathrm{i}_{21 \mathrm{f}}=\mathrm{i}_{21 \mathrm{fc}}=\frac{2}{\mathrm{i}_{\min }+\mathrm{i}_{\max }} \cdot \frac{1}{\eta_{14} \cdot \eta_{21} \eta_{34} \cdot \eta_{23}}$

direction of rotation. This means that the range of possible carrier speeds (positive and negative) is not divided into symmetrical parts $\left(V_{5}^{\prime} \neq V_{5}\right.$ ", see Figure $\left.2 a\right)$. A direct consequence is that also the energy flows from and to the storage spring is not equal.

In order to avoid this unwanted effect already in the design phase, the conventional gear ratio $\mathrm{i}_{23}$ has to be chosen such that the ring gear is rotating exactly in a way that the carrier speed is equal in both cases $\mathrm{i}_{2} 1 \mathrm{~min}$ and $i_{21}$ max. This case is shown in Figure $2 b$, where $V_{5}{ }^{\prime}=V_{5}$ " holds. It should be noticed that the static transmission ratio of the planetary gear $(u)$ is not affected by these considerations.

In Figure 2d, the nonlinear characteristic of i 21 is plotted together with its reciprocal linear function

$$
\frac{1}{i_{21 f}}=\frac{\frac{1}{i_{\text {max }}}+\frac{1}{i_{\text {min }}}}{2}=\frac{i_{\text {min }}+i_{\text {max }}}{2} \quad,\left(i_{\text {min }}=\frac{1}{i_{\text {max }}}\right)
$$

This leads to an additional restriction for the static CVT freewheel transmission ratio for the case of purely kinematical considerations without friction losses:

$$
\mathrm{i}_{21 \mathrm{f}}=\frac{\mathrm{i}_{23}}{\mathrm{u}}=\frac{\mathrm{z}_{1} \cdot \mathrm{z}_{022}}{\mathrm{z}_{3} \cdot \mathrm{z}_{021}}=\frac{2}{\mathrm{i}_{\min }+\mathrm{i}_{\max }} .
$$

The proper choice of i21f taking the above findings into consideration guarantees not only a static carrier $\left(n_{5}=0\right)$ but also a division of the possible carrier speeds into equal parts.

\section{Optimal CVT transmission ratio (i21opt)}

The optimal CVT transmission ratio i21opt is a formulation to compute the necessary CVT control input for a desired torque transfer between storage spring and vehicle. In the control scheme explained later this transmission ratio will act as a feed-forward input to the CVT.

From Figure 1, the desired torque $\mathrm{M} 2$ acting on shaft 2 may be computed as

$$
\mathrm{M}_{2}=\frac{\mathrm{P}_{2}}{\omega_{2}}=\frac{\mathrm{P}_{2_{\mathrm{CVT}}}+\mathrm{P}_{\mathrm{z}_{021}}}{\omega_{2}}
$$

and using the efficiencies of the transmissions it may be expressed by:

$$
\mathrm{M}_{2}=\frac{1}{\omega_{2}}\left(\mathrm{P}_{1} \cdot \eta_{21}+\frac{\mathrm{P}_{3}}{\eta_{23}}\right)
$$

If the power is substituted by the product of force, radius, and rotational speed one gets 


$$
M_{2}=\frac{1}{\omega_{2}}\left(F_{1} \cdot r_{1} \cdot \omega_{1} \cdot \eta_{21}+\frac{F_{3} \cdot r_{3} \cdot \omega_{3}}{\eta_{23}}\right)
$$

Substituting $F_{1}$ and $F_{3}$ by the force acting on the carrier $F_{5}$ together with the respective efficiencies yields

$$
M_{2}=\frac{1}{\omega_{2}}\left(\frac{F_{5} \cdot \eta_{14}}{2} \cdot r_{1} \cdot \omega_{1} \cdot \eta_{21}+\frac{F_{5} \cdot r_{3} \cdot \omega_{3}}{2 \cdot \eta_{34} \cdot \eta_{23}}\right) \text {. }
$$

By expressing the rotational speed ratios with the respective transmission ratios the desired torque $\mathrm{M} 2$ can be finally expressed by

$$
\mathrm{M}_{2}=\underbrace{\frac{\mathrm{F}_{5} \cdot \mathrm{r}_{1}}{2} \cdot \eta_{14} \cdot \eta_{21} \cdot \frac{1}{\mathrm{i}_{21}}}_{\mathrm{M}_{2 \mathrm{CVT}}}+\underbrace{\frac{\mathrm{F}_{5} \cdot \mathrm{r}_{3}}{2 \cdot \mathrm{i}_{23} \cdot \eta_{34} \cdot \eta_{23}}}_{\mathrm{M}_{\mathrm{z} 021}} .
$$

where $M, P, F, r, n, \omega, \eta, i$ are torque, power, spring force, radius, revolutions per minute, rotational speed, efficiency, and transmission ratio (Figure 1).

Solving equation (4) for the optimal transmission ratio of the CVT to ensure this torque one gets accordingly

$$
\mathrm{i}_{21 \mathrm{opt}}\left(\mathrm{M}_{2}, \mathrm{~F}_{5}\right)=\frac{\mathrm{F}_{5} \cdot \mathrm{r}_{1} \cdot \eta_{14} \cdot \eta_{21}}{2\left(\mathrm{M}_{2}-\frac{\mathrm{F}_{5} \cdot \mathrm{r}_{3}}{2 \cdot \mathrm{i}_{23} \cdot \eta_{34} \cdot \eta_{23}}\right)} .
$$

Therefore, depending on the stored spring energy (characterized by $F_{5}$ ) the CVT transmission ratio for a desired torque from the device may be readily computed.

Correction for friction losses: Dynamic and compensated static CVT freewheel transmission ratios (i21d and i21fc)

In this part the purely kinematic considerations of the earlier sections are extended by taking into account the efficiencies of the individual transmissions. This leads to a correction of the static freewheel transmission ratio and a new dynamic freewheel transmission ratio is derived for the purpose of friction compensation.

The dynamic CVT freewheel transmission ratio i21d should only compensate friction losses due to additional inner torques which is especially useful during stretches with constant speeds. In this case the desired torque from the storage device to the drive train is zero $\left(\mathrm{M}_{2}=0\right)$ but the CVT must still transfer a small amount of energy to cover the inner losses.

Using the formulation of i21opt from equation (5) with $\mathrm{M}_{2}=0$ the dynamic CVT freewheel transmission ratio may be formulated as

$$
\mathrm{i}_{21 \mathrm{~d}}=\frac{\mathrm{F}_{5} \cdot \mathrm{r}_{1} \cdot \eta_{14} \cdot \eta_{21}}{2\left(-\frac{\mathrm{F}_{5} \cdot \mathrm{r}_{3}}{2 \cdot \mathrm{i}_{23} \cdot \eta_{34} \cdot \eta_{23}}\right)}=\frac{\mathrm{i}_{23}}{\mathrm{u}} \cdot \eta_{14} \cdot \eta_{21} \cdot \eta_{34} \cdot \eta_{23},
$$

which is now independent of $F_{5}$ and only a function of transmission ratios and efficiencies.

Computing this transmission ratio is not an easy task since the efficiencies of the individual transmissions are highly coupled, nonlinear, varying with speed, torque, and CVT ratio, and also depend on the conditions of the environment (lubrication, temperature, wear). For a practical implementation it would be necessary to determine these relationships experimentally. Nevertheless, i21d needs not be known exactly for proper function of the device, since it should only compensate for friction losses and keep the device from decelerating the drive train.

In any case, a hard limit for maximum energy transfer besides the realizable CVT transmission ratios is the maximum torque in shaft 2 (M2max). Including all efficiencies the maximum torque follows as

$$
\mathrm{M}_{2 \max (\mathrm{imin}, \mathrm{imax})}=\underbrace{\frac{\mathrm{F}_{5} \cdot \mathrm{r}_{1}}{2} \cdot \eta_{14} \cdot \eta_{21} \cdot \frac{1}{i_{\min (\max )}}}_{\mathrm{M}_{2 \mathrm{CVT}}}+\underbrace{\frac{\mathrm{F}_{5} \cdot \mathrm{r}_{3}}{2 \cdot \mathrm{i}_{23} \cdot \eta_{34} \cdot \eta_{23}}}_{\mathrm{M}_{\mathrm{z}_{021}}},(6)
$$

In order to guarantee equal energy flows during accumulation and consumption the following must hold:

$\mathrm{M}_{2(\mathrm{imin})}=-\mathrm{M}_{2(\mathrm{imax})}$

Using equation (6) and rearranging yields

$$
\frac{\mathrm{F}_{5} \cdot \mathrm{r}_{1}}{2} \cdot \eta_{14} \cdot \eta_{21}\left(\frac{1}{\mathrm{i}_{\text {min }}}+\frac{1}{\mathrm{i}_{\max }}\right)=-2 \cdot \frac{\mathrm{F}_{5} \cdot \mathrm{r}_{3}}{2 \cdot \mathrm{i}_{23} \cdot \eta_{34} \cdot \eta_{23}},
$$

where $F_{5}$ may be cancelled. Using the definitions made earlier

$$
\mathrm{u}=-\frac{\mathrm{z}_{3}}{\mathrm{z}_{1}}=-\frac{\mathrm{r}_{3}}{\mathrm{r}_{1}}, \mathrm{i}_{\min }=\frac{1}{\mathrm{i}_{\max }}
$$

and doing some rearranging the following relation results:

$$
\frac{i_{23}}{u}=\frac{2}{i_{\min }+i_{\max }} \cdot \frac{1}{\eta_{14} \cdot \eta_{21} \eta_{34} \cdot \eta_{23}}
$$

This leads to a new static CVT freewheel transmission ratio i21fc which is calculated using the efficiencies of the individual transmissions. The new formulation is analogous to the original one from equation (3) with a correction factor from the individual efficiencies: 


$$
i_{21 f c}=\frac{i_{23}}{u}=\frac{z_{1} \cdot z_{022}}{z_{3} \cdot z_{021}}=\frac{2}{i_{\text {min }}+i_{\max }} \cdot \frac{1}{\eta_{14} \cdot \eta_{21} \eta_{34} \cdot \eta_{23}}
$$

And the dynamic CVT freewheel transmission ratio i21d is simply derived by multiplying the above result with the efficiencies of the transmissions:

$$
\mathrm{i}_{21 \mathrm{~d}}=\mathrm{i}_{21 \mathrm{fc}} \cdot \eta_{14} \cdot \eta_{21} \cdot \eta_{34} \cdot \eta_{23}
$$

The conditions during a change in the transmission ratio (i23) and consequently the change in velocity $V_{3}$ taking into account the efficiencies can be seen in Figure 2c.

The reason for an increase in $V_{5}$ ' compared to $V_{5}$ " will be illustrated using the energy flow charts depicted in Figure 3: Two charts are given for the case of energy accumulation (above) and energy consumption (below). The main energy flows may be summarized as follows:

- During braking energy is accumulated by a main power flow through the conventional gear, ring gear, planet gear, carrier, and into the storage spring.
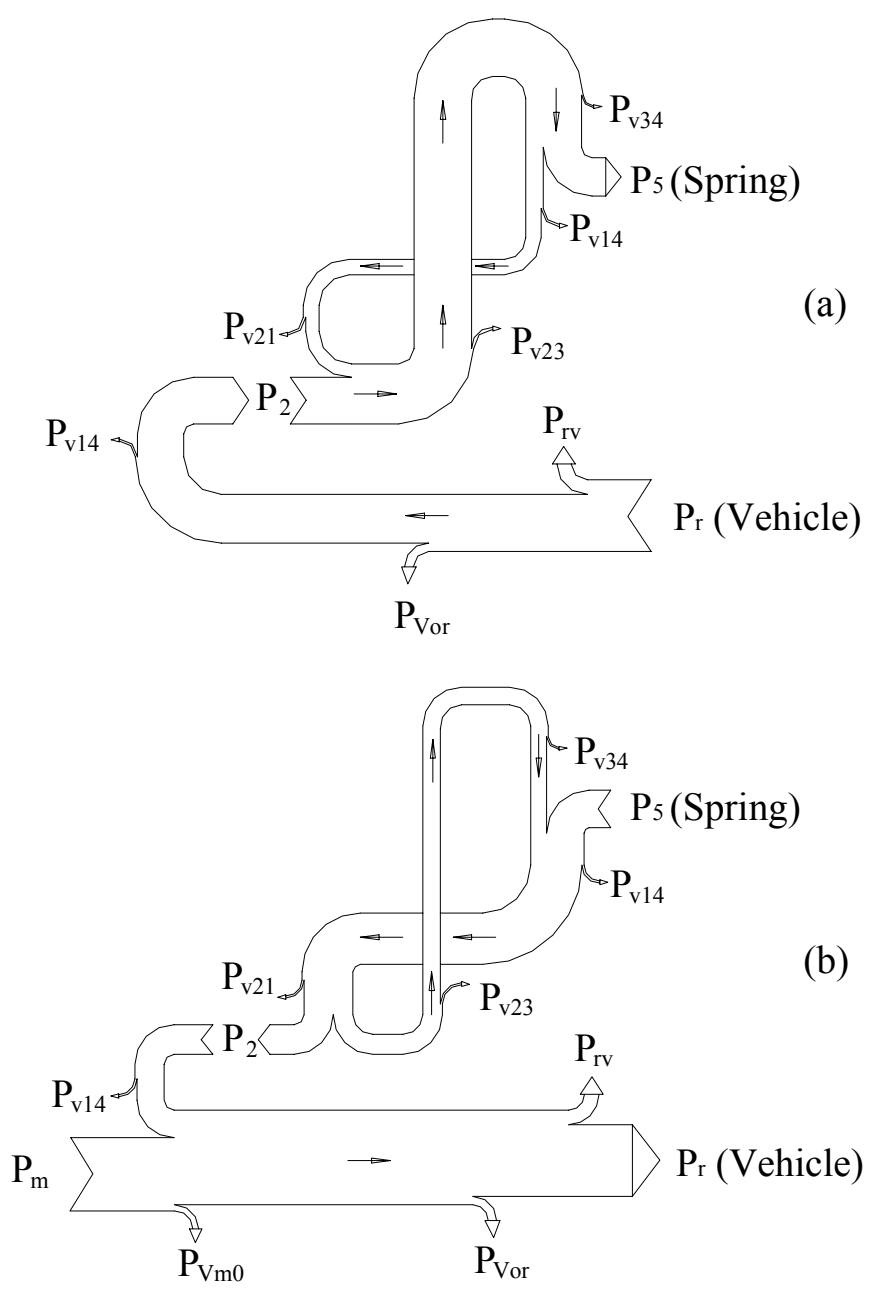

Figure 3: Energy flow chart during accumulation (a) and consumption (b)
- The consumption of stored spring energy for acceleration causes the energy to flow from the storage spring into the carrier, planetary gear, ring gear, and through the CVT into the drive train.

Because the CVT has a smaller efficiency than the conventional gear more energy is dissipated during acceleration compared to energy accumulation during braking.

It is important to notice that this transmission ratio may not be realizable due to geometric restrictions. However, for a mechanical efficient design it is imperative to account for an optimal splitting of the range of the CVT ratios.

If no losses are considered the dynamic and the static freewheel ratios must be equal:

$\mathrm{i}_{21 \mathrm{~d}}=\mathrm{i}_{21 \mathrm{fc}}$

For longer stretches of travel with constant speed the storage mechanism should be completely separated from the drive train in order to conserve the stored spring energy and to minimize friction losses.

\section{CONTROL STRATEGY}

Control strategies for hybrid vehicles are already established and implemented in consumer cars [8], and detailed control algorithms have been published for hybrid electric vehicles (HEV) [9]. Although these strategies were developed for hybrid vehicles with a combustion engine combined with an electric motor/generator they could be readily applied to the purely mechanical storage device presented in this paper. If the electric part is replaced by the mechanical storage device and the control input is tailored to adjust the CVT transmission ratio the control concept may be adopted with only small changes in hardware specific areas.

The control strategy proposed here is not to be taken as a fully developed and optimized concept, but it is rather a simple scheme to demonstrate the basic functionality of a suitable control strategy.

The CVT transmission ratio $\mathrm{i}_{21}$ constitutes the control input. It is computed from several input variables (Figure 4):

- Drivers request for acceleration or deceleration,

- vehicle velocity $V$ (or kinetic energy Ek),

- current amount of energy stored in the spring Ep,

- brake and engine state 


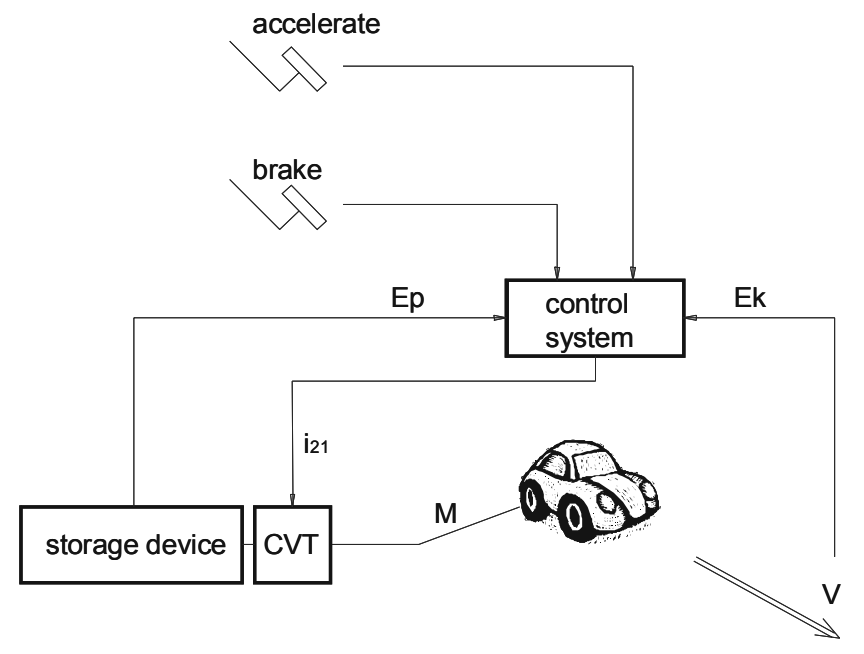

Figure 4: Principle of control

The simplified control strategy used for the simulation example described later is given in Figure 5: First, the vehicle velocity is retrieved, and then the drivers' intentions to either brake or accelerate are detected. In both cases prior to an adjustment of the CVT the state of the storage spring is checked:

In case the driver wants to decelerate, the storage spring must still have capacity to store more energy; if the result of this check is positive a control loop is activated which ensures that as much energy as possible is accumulated in the storage spring, while the deceleration of the vehicle is in agreement with the drivers request. The control input to the CVT is then chosen to be the freewheel transmission ratio (feed-forward) plus a corrective input from a closed-loop regulator (feed-back).

If the maximum deceleration in this mode is still too small, the main brakes are automatically engaged to ensure proper braking. If the storage device does not have any additional capacity then a conventional braking maneuver is performed.

If an acceleration of the vehicle is wanted, the energy consumption from the storage device has first priority. Only if there is no more energy left in the spring or if a larger acceleration is requested the engine delivers additional torque.

Remaining energy in the storage device is unwanted since it produces higher losses. Nevertheless, if the spring still contains a large amount of energy during constant velocity travel the device may be separated from the drive train using a simple clutch. In this way the energy may be conserved virtually without loss for an arbitrarily long stretch of time.

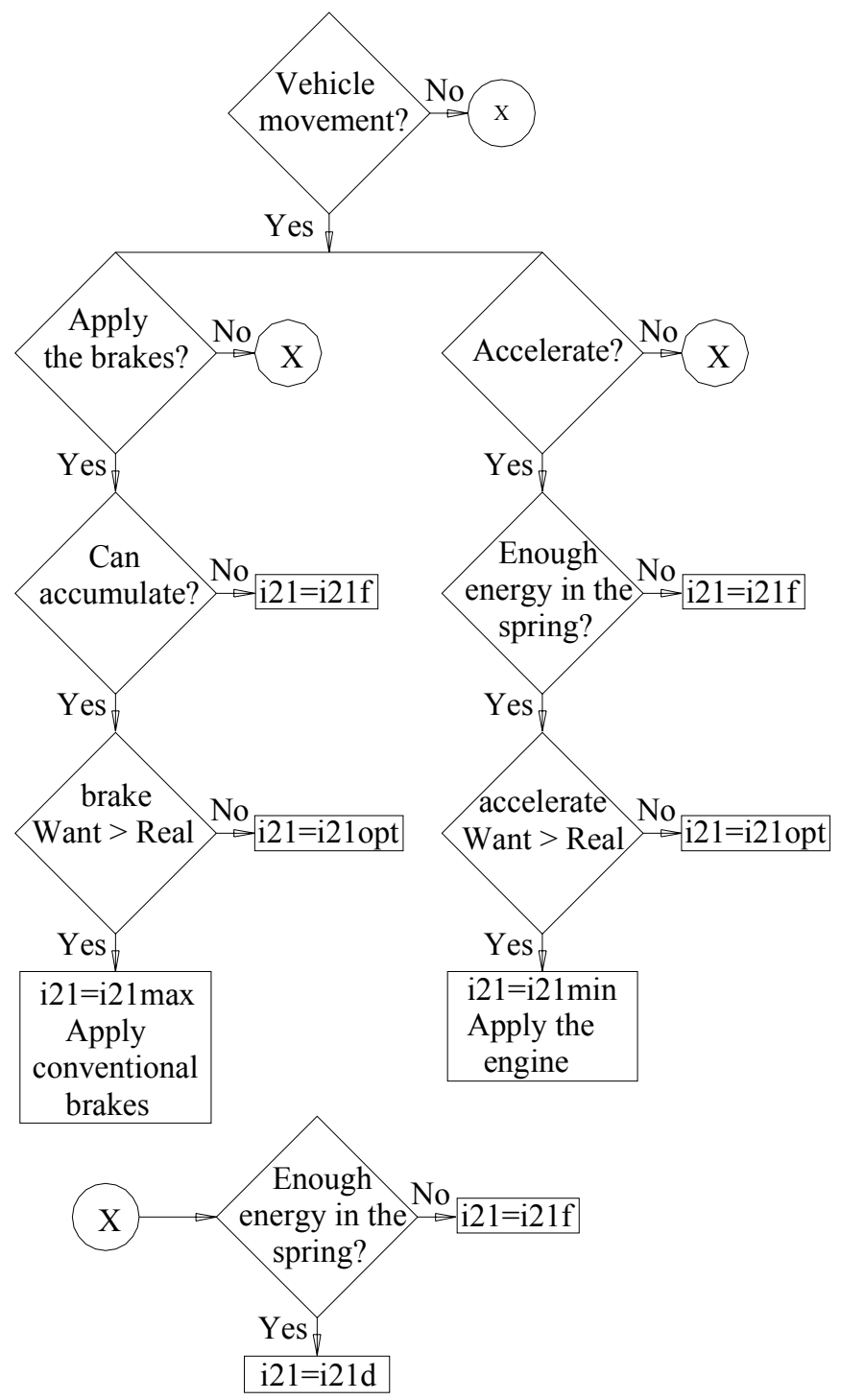

Figure 5: Control strategy for CVT transmission ratio

\section{SIMULATION}

A simulation of a simple longitudinal vehicle model was performed to study the potential of the proposed concept. Using a lumped mass model the energy savings for different test cycles were computed and compared.

The lumped mass model consisted of the vehicle mass, the rotational inertias of wheels, drive train, and engine plus gear box. Since elastic couplings between these masses have been neglected, one reduced mass at rotational speed $n_{2}$ can be utilized. The simulation was performed in in MATLAB ${ }^{\circledR}$ Simulink where both the dynamic vehicle model and the control algorithm could be implemented easily.

The simulation model has not been not been validated by measured data. However, model data and structure have 
been chosen quite conservative and the dynamic behavior of the model is absolutely plausible. The numerical results should therefore not be taken as absolute numbers but rather as an indicator of what performance the new concept is capable of.

\section{Data and simplifications of the model}

The following assumptions were made for the simulation model:

Vehicle:

- Vehicle mass (including the storage device) is constant at $m=1500 \mathrm{~kg}$

- The road is level.

- The vehicle is divided into 5 main components: Engine, clutch, gear box, differential gears and wheels.

- Clutch operations and gear shifting have not been investigated since they do not significantly affect the energy balance during velocity changes.

- Efficiency of gear box $=98 \%$.

- Efficiency of differential gear $=95 \%$.

- A reduction of all rotating masses to shaft 2 is performed.

- Rotational inertia of the shafts was neglected.

Transmissions in the storage device:

- The efficiency of the CVT is assumed to be constant $=85 \%$.

- $\quad$ All other gear efficiencies $=98 \%$.

- Like above, all rotating masses are reduced to shaft 2.

- The rate of change in the CVT ratio is only a function of the rotational speed at the input shaft.

- A medium CVT angular velocity $\omega_{21}=\frac{\omega_{2}+\omega_{1}}{2}$ is computed, and a change through the half range of the CVT from $i_{21}=1$ to $i_{21}=i_{\max (\min )}$ is assumed to last 8 full revolutions.

- Maximum CVT ratio: $\quad \mathrm{i}_{\max }=2.5$

- Minimum CVT ratio:

$$
\mathrm{i}_{\text {min }}=\frac{1}{i_{\text {max }}}=0.4
$$

\section{Storage spring:}

The storage spring is assumed to be a spiral spring with the following characteristics [11]:

- Ideal spring (no hysteresis).

- $\quad$ Mass of spring $=59 \mathrm{~kg}$.

- $\quad$ Maximum stored energy $=30 \mathrm{~kJ}$.

- Max. torque $=676 \mathrm{Nm}$.

- Maximum revolutions $=6.2 \mathrm{rev}$.
- Width of spring= $0.083 \mathrm{~m}$.

- Diameter of spring housing $=0.51 \mathrm{~m}$.

- Energy density of device = app. $0.12 \mathrm{Wh} / \mathrm{kg}$

- The power density is difficult to define, since the power output of the device strongly depends on the vehicle speed and the CVT transmission ratio. Nevertheless, depending on the vehicle state high power outputs are possible.

Additional data:

- $\quad \mathrm{i}_{21 \mathrm{f}}=0.9783$

- $\quad \mathrm{i}_{21 \mathrm{~d}}=0.7826$

- $\quad \mathrm{Z}_{1}=10$

- $\mathrm{z}_{3}=46$

- $\mathrm{i}_{23}=-4.5$

\section{Simulation Results}

In Figure 6 the results for a test cycle which changes 3 times per minute between $1 \mathrm{~m} / \mathrm{s}$ and $6 \mathrm{~m} / \mathrm{s}$ at a rate of $1 \mathrm{~m} / \mathrm{s}^{2}$ are plotted. The solid line is the engine power plotted versus time, while the dashed line is the total power available for acceleration. The arrows point to the areas where a clear energy saving can be seen due to the contribution of the storage device. In the transient range the engine feeds a small amount of energy into the device which is due to a non-optimized controller.

The second test cycle (Figure 7) includes velocity changes from $0 \mathrm{~km} / \mathrm{h}$ to $36 \mathrm{~km} / \mathrm{h}$, with an acceleration of $\pm 1 \mathrm{~m} / \mathrm{s}^{2}$, and seven cycles per kilometer. This cycle causes the spring to be fully wound up after each braking maneuver. In this simulation an energy saving of $E_{\text {Diff }}=$ $53.3 \mathrm{~kJ} / \mathrm{km}$ resulted for the vehicle with storage device. The additional mass of the storage device and the slightly worse efficiency of the transmissions are already accounted for in this result.

Saved energy was referenced to shaft 2 in the simulations due to the mass and rotational inertia reduction. It is therefore possible to compute the equivalent additional fuel consumption taking into account the efficiency of the combustion engine and the drive train.

The energy saving can be computed using the efficiencies and the energy difference between a vehicle equipped with the device and a conventional vehicle:

$\mathrm{E}_{\mathrm{M}}=\frac{\mathrm{E}_{\text {Diff }}}{\eta_{\mathrm{M}} \cdot \eta_{\mathrm{m} 0} \cdot \eta_{\mathrm{i} 02}}$ 


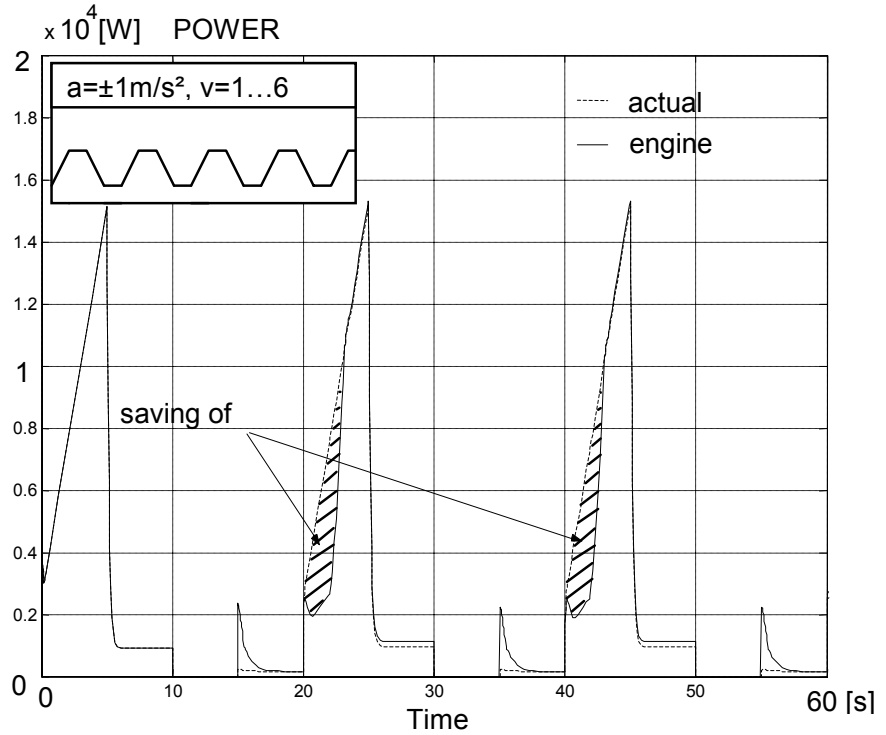

Figure 6: Area of energy saving

The saving of fuel per $100 \mathrm{~km}$ can be calculated using the net calorific value $\mathrm{Hu}$ :

$\mathrm{m}_{\text {Diff }}=\frac{\mathrm{E}_{\mathrm{M}} \cdot 100 \mathrm{~km}}{\mathrm{Hu}}=\frac{199.9 \cdot 10^{3} \cdot 100}{42 \cdot 10^{6}}=0.476 \mathrm{~kg}$

...(per $100 \mathrm{~km})$

The above result was computed for a constant CVTefficiency of $85 \%$. However, the true efficiency of the CVT depends on the torque load, the transmission ratio and rotational speed and can be higher. For a constant efficiency of $90 \%$ a fuel saving of $0.724 \mathrm{~kg} / 100 \mathrm{~km}$ will result while for an efficiency of $80 \%$ the saving will be $0.235 \mathrm{~kg} / 100 \mathrm{~km}$. The highest efficiency of the CVT will be achieved during acceleration from a stand-still.

This means that a vehicle equipped with the storage device will save $0.476 \mathrm{~kg}$ fuel per $100 \mathrm{~km}$ in a typical urban stop and go situation. It should be noticed, that an overall efficiency of the engine of $20 \%$ is very optimistic for this traffic situation, and therefore, the more acceleration/deceleration cycles per $\mathrm{km}$ occur the larger even a specific energy saving will be.

The direct comparison to a HEV would be important, however, no numbers on the efficiency of regenerative braking could be found in an extensive literature search. A Toyota Prius reclaims approximately $30 \%$ of its kinetic energy during braking, but due to losses only part of this energy will be transformed into kinetic energy during the next acceleration. For HEVs without a separate generator, these numbers will be considerable smaller. Additionally, due to restrictions in the battery charging voltage a HEV is not able to accept all the regenerative braking energy especially during strong decelerations [10].

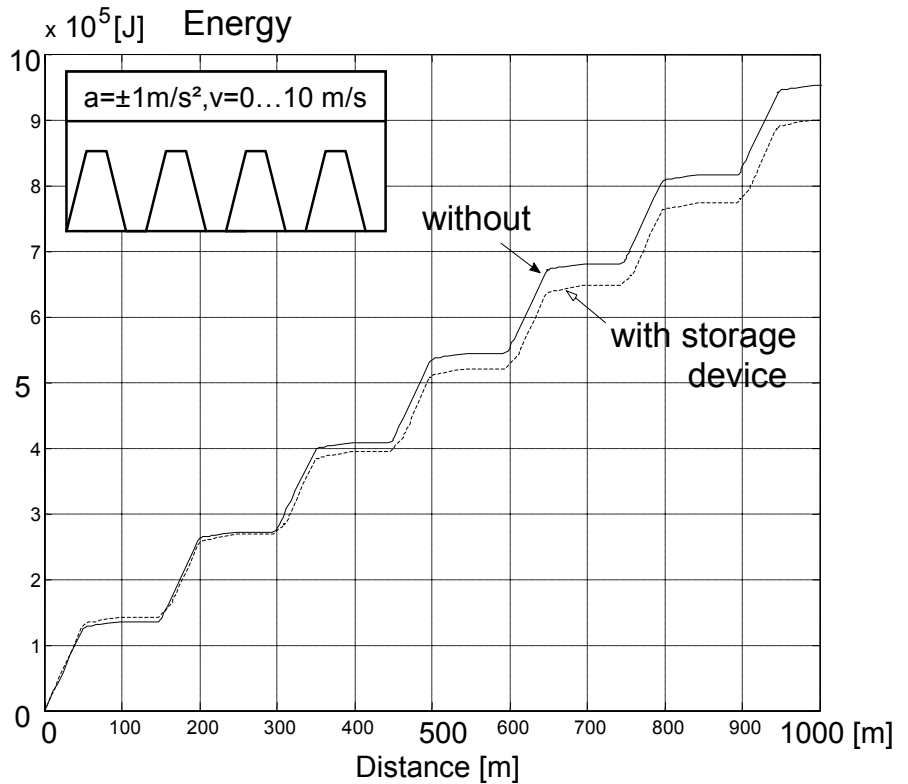

Figure 7: Energy consumption with- and without storage device

\section{CONCLUSION}

In this paper a novel purely mechanical energy storage device for regenerative braking has been presented. The mechanism consists of a combined CVT, conventional, and planetary gear together with a spiral spring for energy storage. During deceleration part of the kinetic energy is fed into the spring and during acceleration this energy may be used to aid the engine. The central part of the mechanism is the interaction of the individual transmissions which enable the storage device to work efficiently under a wide range of driving conditions.

The principles of the mechanism are explained and the important relations for transmission ratios and torques are derived. Also practical and constructive considerations are mentioned.

In order to relieve the driver from any additional tasks an automated control scheme is presented, which guarantees correct operation of the device under all driving conditions. This control scheme decides automatically how energy should be distributed or dissipated during acceleration or braking. The control variable is the CVT transmission ratio, which decides if energy is accumulated in the storage spring or consumed by the propulsion of the vehicle.

In a computer simulation several test cycles were performed. The comparison of vehicle models with or without the proposed device clearly shows that a positive effect on energy saving is achieved. These energy savings are largest if frequent stop and go cycles are passed through. 
The applicability of this device to consumer vehicles strongly depends on the legislative conditions and the technological improvements of the CVT and the spring. The efficiency of the CVT as well as the weight of the spring strongly affect the overall efficiency of the device. It is also obvious that commercial vehicles like small buses, fork lifts or similar vehicles could be possible applications. In order to achieve maximal efficiency the size of the spring and consequently its maximum energy storage capacity should be tailored to the typical drive cycle of the vehicle. However, due to the limited energy storing capacity the proposed concept is not a competitor to HEVs with their typical ability to travel long distances using the electric drive. It is rather an attractive solution for frequent stop-and-go cycles at moderate speed levels.

Due to the conservative simplifications in the model and a standard controller design without optimization there is still a lot of potential to refine the parameters of the concept and yield even better results for the specific energy saving with the proposed device.

\section{REFERENCES}

1. The 14th International Electric Vehicle Symposium,Orlando (USA), 1997, „Electric Motor for TETLEI Program (Brite Euram)“/ H.-J. SchmidtBrücken, C. Fournier.

2. Hybridantriebe : Tagung Garching, 25. und 26. Februar 1999 / VDI-Gesellschaft Entwicklung, Konstruktion, Vertrieb . - Düsseldorf : VDI-Verl. , 1999 . - 426 S. . - ( VDI-Berichte ; 1459 ). - ISBN 318-091459-9

3. Dispositif pour emmagasiner et resituer de l'energie et application a la propulsion d'un vehicule/ Bommer Raymond: Patent FR 2278945 A1, 13.02.1976

4. Regenerative braking device/ Lyle O. Hoppie: U.S. Patent 4,310,079, 01.12.1982
5. Vorrichtung zur Bremsenergiespeicherung in Spiralfedern: Arabadjiev Dimitre: Patentschrift DD 292306 A5, 08.12.1986

6. Vehicle drive assembly: Van Druten, Roell Marie: European Patent EP 0952023 A1, 27.10.1999

7. Zahnradgetriebe : Grundlagen und Konstruktion der Vorgelege- und Planetenradgetriebe / Johannes Looman . - Berlin [u.a.] : Springer , 1970 . - VII, 287 S. . - ( Konstruktionsbücher ; 26 )

8. Sasaki S., IEEE Symposium on Power Semiconductor Devices \& ICs (ISPSD), Kyoto, Japan, June 3-6, 1998, pp 17-22: “Toyota's newly developed hybrid powertrain"

9. Xiaoling He and Jeffrey W. Hodgson: "Modeling and Simulation for Hybrid Electric Vehicles - Part I: Modeling", IEEE Transactions on Intelligent Transportation Systems, Vol. 3, No. 4, Dec. 2002

10. Frank A. Fleming, Philip Shumard, Blake Dickinson: "Rapid Recharge Capability of Valve Regulated Lead Acid Batteries for EV \& HEV Applications", White Paper from Hawker North Amerika, EnerSys Inc., http://www.hepi.com/papers.htm

11. Handbuch Federn : Berechnung und Gestaltung im Maschinen- und Gerätebau / Manfred Meissner ; Klaus Wanke ; [Hans-Jürgen Schorcht] . - 2., bearb. Aufl. . - Berlin [u.a.] : Verl. Technik, 1993 . - 324 S. . - ISBN 3-341-01087-4 Gb. H.-J. Schmidt-Brücken, C. Fournier.

\section{CONTACT}

Tchobansky Latchezar

Institute for Machine and Process-Automation

Vienna University of Technology

Gusshausstrasse 27-29 / 328

A-1040 Vienna

Austria

Fax: +4315880132899

E-mail: boko@rosko.net 